\title{
OPNET: A Solution for Software and Hardware Networking
}

\author{
Shivaraj Hublikar ${ }^{1}$, Arun L. Kakhandki ${ }^{2}$ Anil M. Kabbur ${ }^{3}$, Priyatamkumar ${ }^{4}$ \\ Department of Electronics and Communication Engineering, \\ B. V. Bhoomaraddi College of Engineering \& Technology, Hubli, Karnataka, India \\ ${ }^{1}$ shivaraj@bvb.edu \\ 2arun@bvb.edu \\ 3amkabbur@bvb.edu \\ 4priyatam@bvb.edu
}

\begin{abstract}
OPNET (Optimized Network Engineering Tool) tool introduces the innovative techniques for teaching and learning computer networking and hardware [1]. This tool helps teachers to succeed in dealing with the cost complication and space restriction, in teaching networking and hardware ideas and increase student's participation in flexible learning activities. It helps to starts in with a good comprehension of computer networking and hardware forming necessary basic concepts which are essential. There after it provides lab activities designed to enhance teaching and learning various aspects of computer networking. It is known fact that in computer education, understanding of computer communication networking and hardware concepts are very important topics, but many students appear to think the subject rather technical and too long. Thus, it is necessary to improve learning environment and guide students in exactly motivated with susceptibility for forming a essential core for the concepts. The objective of using OpNet to having existed a bridge between theory and practice in computer networking and hardware field by adopting various approaches, it also cover a broad range of learning about wired and cellular networking, hardware, and custom, but it give special importance to interactive simulative activities in order to motivate students to learning real-world examples. The lab experiments help to enhance knowledge and student's practical skills.
\end{abstract}

Key words: OPNET tool, computer networking, hardware, teaching, learning, Virtual Network Environment (VNE)

\footnotetext{
Shivaraj Hublikar

Department of E\&C Engineering,

BVBCET, Hubli, Karnataka, India

shivaraj@bvb.edu
}

\section{Introduction: The problem}

It has been widely known that practical design and realization is one of the vital skills that students should obtain to become capable computer networking engineers. Online community helps to maintain such a state. Constant relationships creates contract to fulfill group work in networking domain. At instant many associations creating environment of teaching and learning the networking technologies. Foodstuffs are given by OpNet Modeler. We used OpNet simulation tool Academic Edition 17.5 (freeware) for all the experiments/activities we conducted [2]. OpNet gave us foolproof Graphical User Interface (GUI). By assist of the OpNet tool we are able to comfortably boost persistence in the different topologies \& configurations and examine the system distinctiveness and scheme. OpNet includes a many mock-up genre which empower us to the mirroring of current hardware scenarios and OSI-ISO custom. By this we achieve great extent of effort to reduce the cost. Now the question is how effectively we can follow different teaching approaches? We know so many closely connected concepts are need much effort to really hold firmly only in theory. Lectures on one's own do not achieve the skillful performance and practical's - laboratory assignments - must move above common programming assignments. In this approach, a practice of communications protocol is a best workout, and it gives hands on with this orientation of the technology and make it accessible for classroom use. There is an argument of cost. Many institutes (not all) presently may not willing to invest capital on networking labs to occupy the closely associated costly hardware setup along with required software support. Moreover true systems may compass not simply a lab but instead whole structures or even different worldwide areas. Most systems are fabricated to suit the needs of a solitary association or gathering. Internetworking is an innovation that obliges numerous, various, fundamental 
equipment by giving the method for interconnecting heterogeneous systems. Comparatively exclusively dedication need for different users. we may face difficulty here: It is upscale of laboratory setup and other hand pedagogy restrictions. It is challenge and tough task to brainstorm organizations to create a small office networks within a campus with definite workplace.

\section{Solutions}

There are few applicable clarifications that would explain use of traditional and also those of non-traditional (distance) networking students. The main answer of curiosity here is network scenario creation and simulation of the same, particularly OpNet goes to be the main focus [6]. Network creation and simulation software could be used to model networks. Varying with respect to scale is possible. Students learn by creating virtual networks and devices of all kinds and are not limited by network hardware availability interest. Virtual network allows to create different ways - for example routers can be added or subtracted, procedure switched around or altered, similarly servers can add or subtract or removed- any alteration can done easily. The stuff of various modifications and distinct compositions can then be cordially and significantly checked. Mainly OpNet grant us effective inspect and assemble advantageous data about different virtual scenarios created from it. OpNet privilege not only the building of a virtual network in software but it helps to achieve powerful investigating the network. We have many approaches in to create a OpNet virtual network environment (VNE). The highest profit can be obtained by using OpNet's graphical user interface plan. The natural policy is to prefer applicable hardware elements. Nodes, hubs, switches, server, and routers then establish connectivity to obtain benefit from topology [7].

\section{Modeling a network}

For different activities/experiments, we followed the some of the important and typical steps. In general we realized the steps to be follow, to achieve the desired scenarios.

Foot prints $(\mathrm{F})$ to be followed:

F1: Network topology creation.

F2: Scenario Creation.

F3: Assigning statistics based on requirement(s) or expectation from scenario(s).

F4: Simulation (with simulation time assigning).

F5: Result(s) / analysis.

Using scenarios in a model helps us to split a large simulation work into smaller components.

\section{List of activities we conducted}

I. Create a scenario to simulate and analyze the performance of the ethernet network for office scale with size $100 * 100 \mathrm{~m}$.
II. Create a scenario to examine the performance of token ring.

III. Create a scenario to examine the performance of LANs.

IV. Optimization and performance evaluation for a designed network.

V. $\quad$ Experiment to set up an ATM network that carries voice, email and FTP applications and evaluate the performance of these applications by the choice of adaptation layer.

VI. Simulate and examine the effect of different queuing disciplines in packet delivery and delay for different services.

VII. Configure and analyze Routing information protocol [RIP].

VIII. Configure and analyze the performance of OSPF (Open Shortest Path First) routing protocol.

\section{Build a network, choose statistics, run simulation and analyze the results - an example}

\subsection{Creating project}

5.1.1 Entering scenario wizard with following specifications:

Table 1

\begin{tabular}{|l|l|l|}
\hline Initial Topology & $\begin{array}{l}\text { Create Empty } \\
\text { Scenario }\end{array}$ & Click Next \\
\hline Network Scale & Office & Click Next \\
\hline Specify the Size & $100 \mathrm{~m}^{*} 100 \mathrm{~m}$ & Click Next \\
\hline Model family & Ethernet & Click Next \\
\hline Review check values & & Click OK \\
\hline
\end{tabular}

5.1.2 Network topology creation using rapid configuration: Construction of Network:

Table 2

\begin{tabular}{|l|l|}
\hline \multicolumn{1}{|c|}{$\begin{array}{c}\text { Node Model } \\
\text { (Centrally assigned })\end{array}$} & \multicolumn{1}{|c|}{$\begin{array}{c}\text { Ethernet16_hub (Ethernet } \\
\text { family consideration) }\end{array}$} \\
\hline Node model (Periphery) & Ethernet_wkstn \\
\hline Link Model & 10 BaseT \\
\hline Number & 5 \\
\hline $\begin{array}{l}\text { Center } \\
\text { Radial distance }\end{array}$ & $\begin{array}{l}\mathrm{X} \text { axis }=25 \text { Y axis }=25 \\
\text { Consider 20 }\end{array}$ \\
\hline
\end{tabular}

Visual 1 


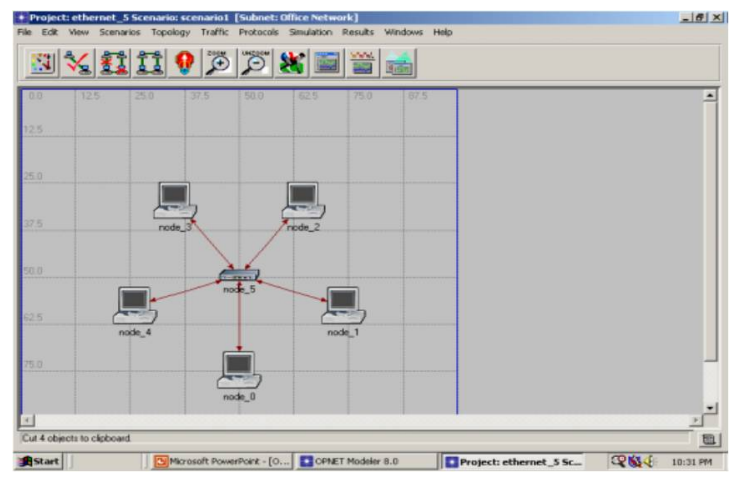

Fig.1 Network Topology Rapid Configuration.

\subsubsection{Adding Components}

Object configurations:

1) Drag Application Config object into the Project workspace.

2) Place into workspace.

3) Minimize the object palette.

Visual 2

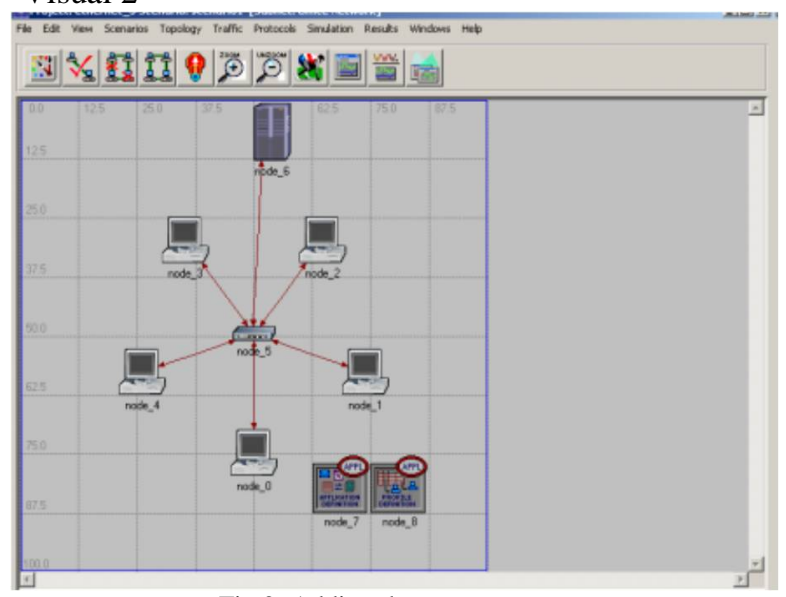

Fig.2 Adding the components.

\subsubsection{Editing Attributes}

I. Change the nature by editing attributes of the object.

II. Click on the object (Right click) say edit to change the properties

III. Click on Edit attributes option.

5.1.5 Assigning Traffic to network.

1) Define_application to Default

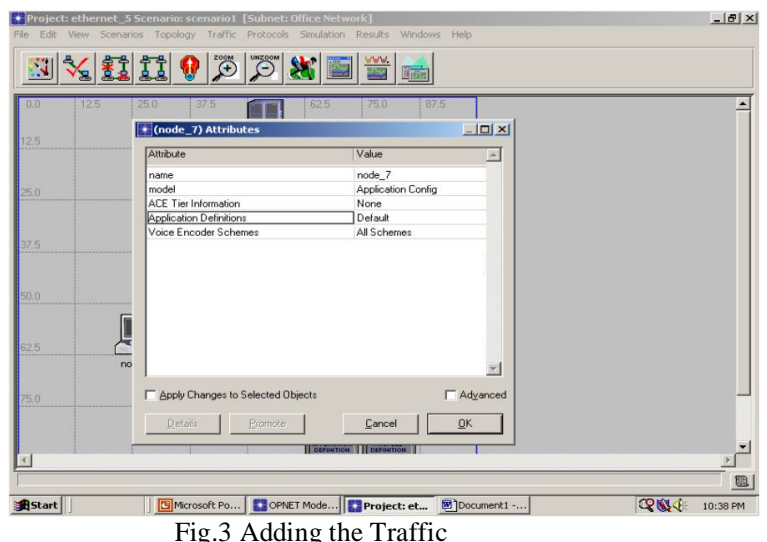

Fig.3 Adding the Traffic

1) Profile Configuration:

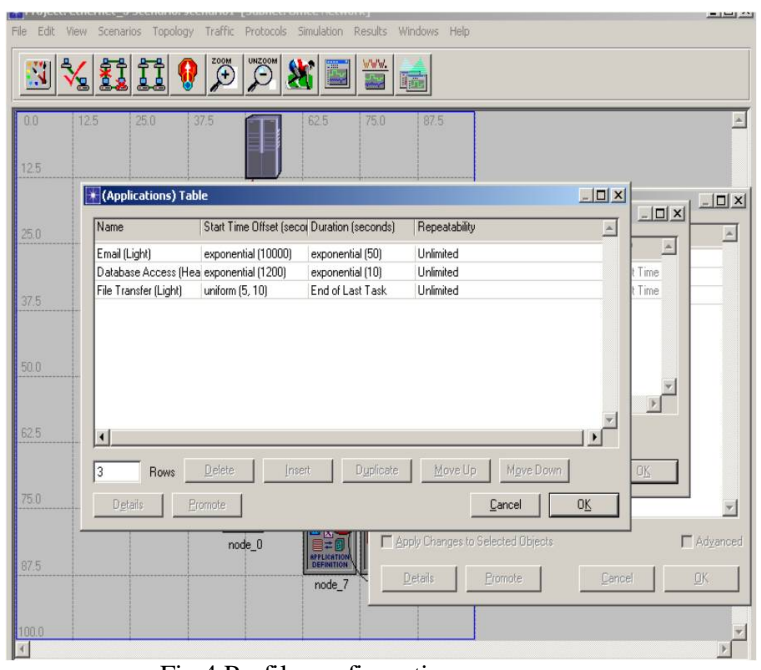

Fig.4 Profile configuration

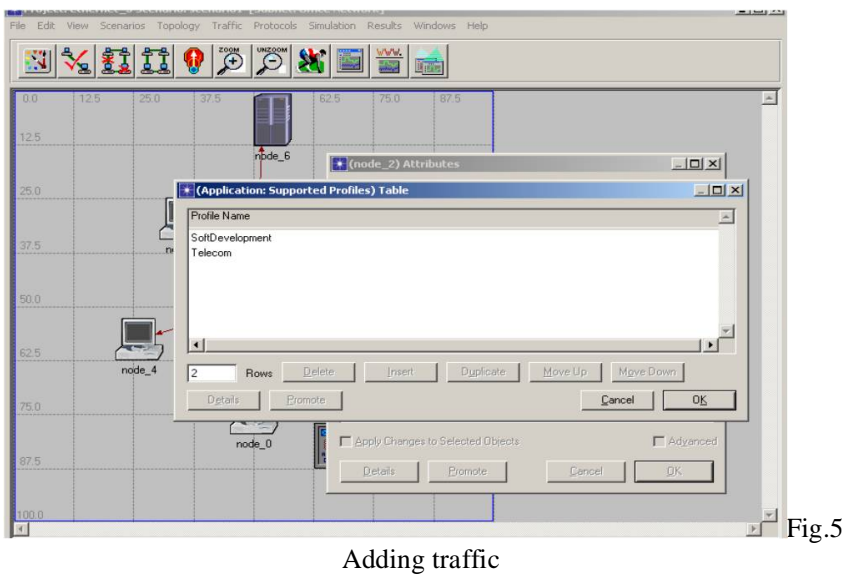

To establish a server connectivity, click on Applications and select supported services and choose ALL. 
Visual 3

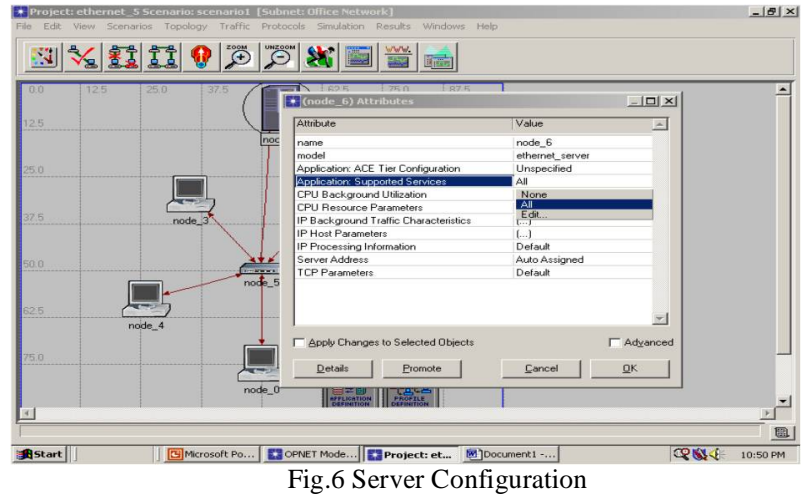

5.1.6 Parameters to be considered for analysis : There are two approaches to collect statistics

1) Individual (Object specific)

2) Global (Entire Network taken into account)

After adding Server Statistics and Network Statistics:

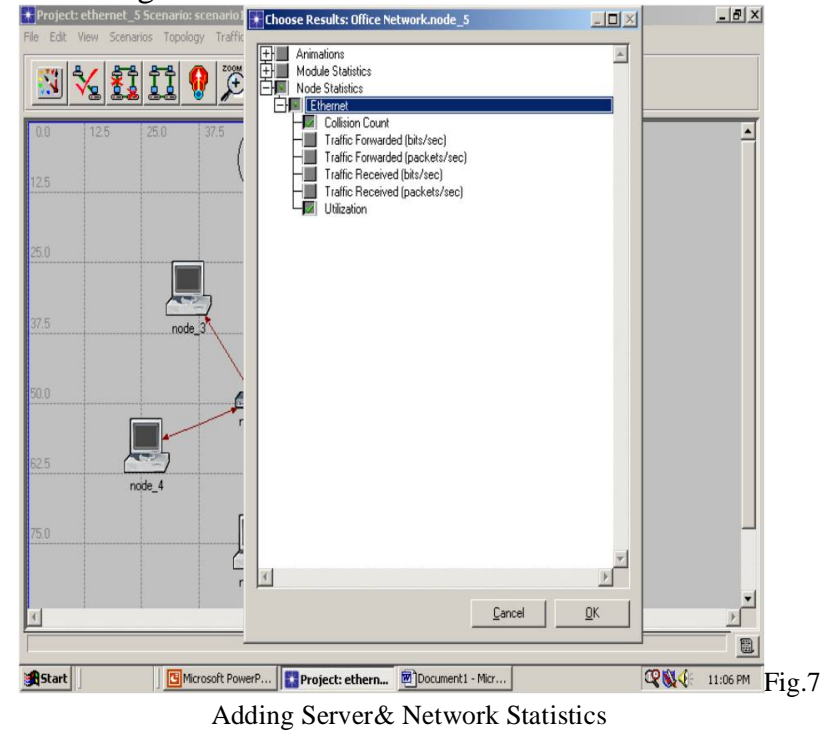

After adding Global Statistics:

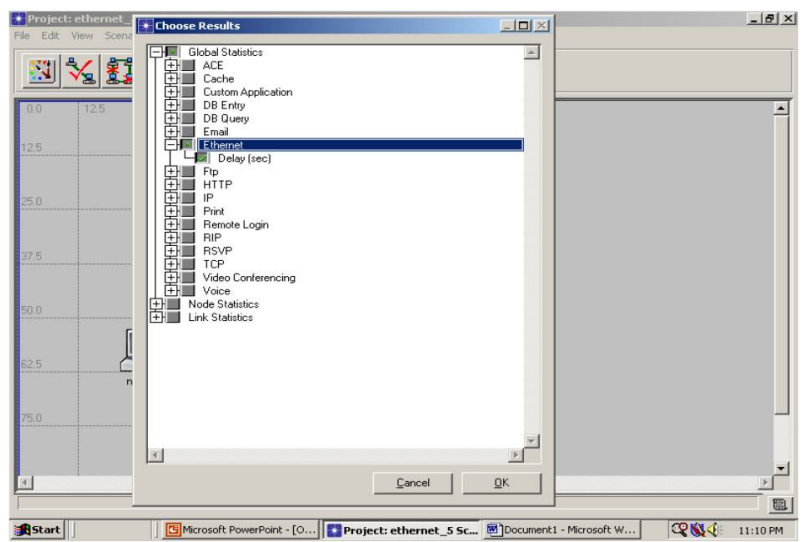

Fig.8 Adding Global Statistics

\subsubsection{Simulation:}

Now we have created the scenario with specification orientation, assigned traffic, statistics for analysis and save the project to simulate.

F1: Go to the DES menu.

F2: Choose configure / Run option. (Ctrl+R)

F3: Assign Simulation duration

\subsubsection{Simulation Results:}

Now our job is to check the obtained statistics are matching with our desired scenario. With respect to traffic assigned, components considerations, network scale and processor speed it may vary.

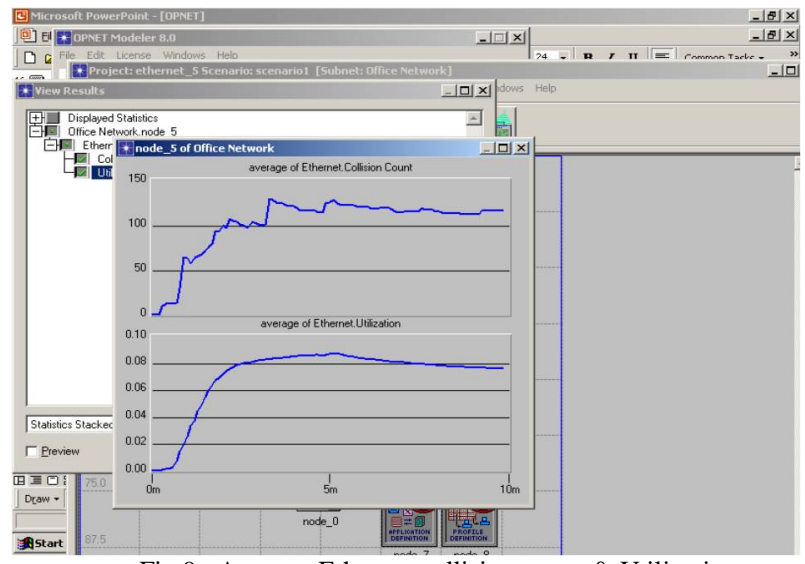

Fig.9 Average Ethernet collision count \& Utilization 


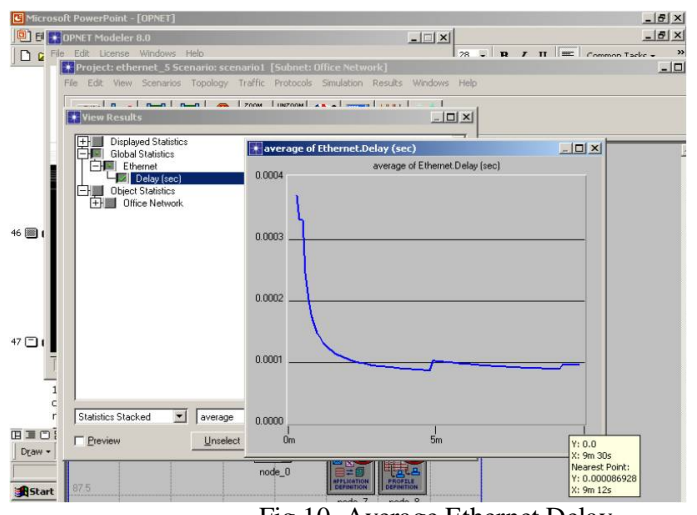

Fig.10 Average Ethernet Delay

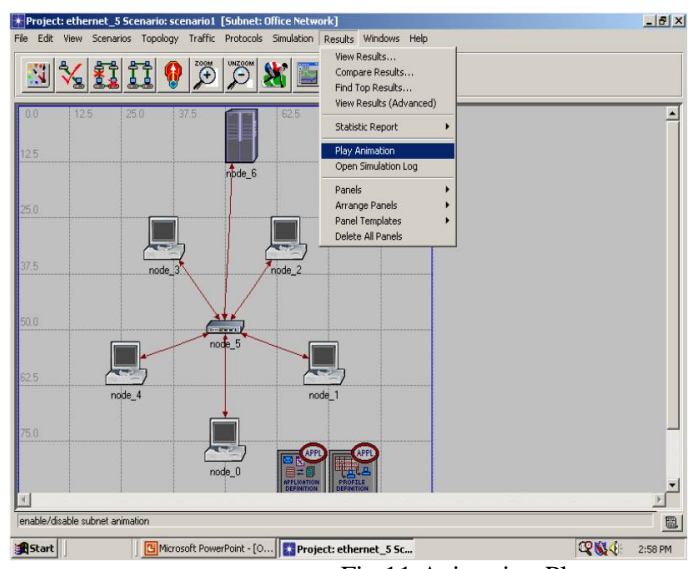

Fig.11 Animation Play

\subsubsection{Find if any errors/ Debug:}

i) Simulation log is best suited for debugging.

ii) It needs time and effort to overcome if errors are more than expected!!

\section{Outcome(s):}

i) Effective usage of modern tools.

ii) Lifelong learning.

\section{Preliminary Assessment:}

Direct measurements: It includes,

i) Conducting exams

ii) Giving different scenario to design and simulation for each student.

Evaluate each student for each assignment/activity (CIE)

Indirect measurement: It includes,

i) Conducting survey (knowledge based and skill based)

ii) Collecting feedback
Table 3

\begin{tabular}{|l|l|}
\hline \multicolumn{1}{|c|}{ Knowledge } & \multicolumn{1}{c|}{ Skills } \\
\hline Network design process & General computing skills \\
\hline Network simulation & Communication skills \\
\hline $\begin{array}{l}\text { Network performance } \\
\text { analysis }\end{array}$ & Math skills \\
\hline $\begin{array}{l}\text { Network topology (bus, } \\
\text { star, etc.) }\end{array}$ & $\begin{array}{l}\text { Computer network design skills } \\
\text { performance using simulations }\end{array}$ \\
\hline Internet routing & $\begin{array}{l}\text { Ability to use OpNet to explore } \\
\text { and nearn new network } \\
\text { protocols }\end{array}$ \\
\hline $\begin{array}{l}\text { TCP flow control and } \\
\text { congestion } \\
\text { control }\end{array}$ & Usage of modern tools. \\
\hline OpNet Software &
\end{tabular}

Data collected so far is the pre and post surveys. Figure 12 and 13 shows the analysis results..

Rating: 1-"None", 2- "Poor", 3- "Fair", 4- "Good", 5"Excellent".

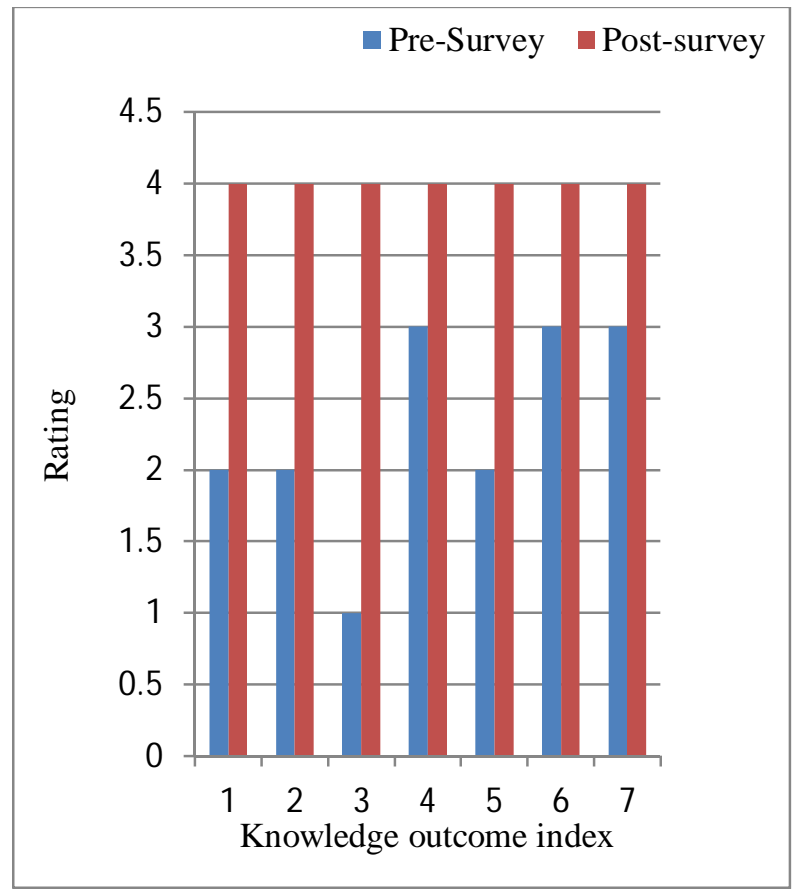

Fig.12 Knowledge outcome index 


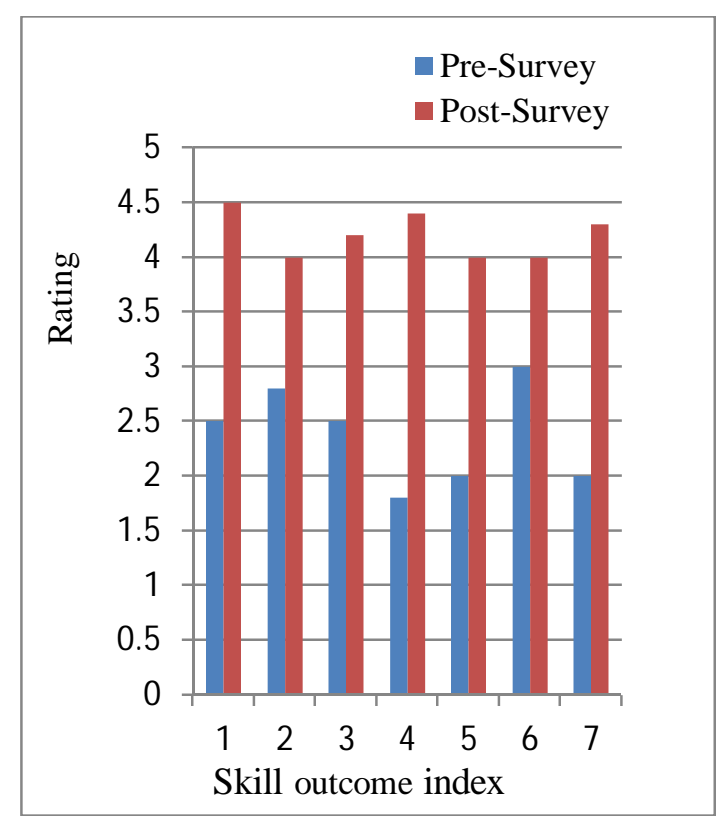

Fig.13 Skill outcome index

\section{Conclusion :}

In this paper we have presented an overview of how, we effectively used network simulation tool to enhance student learning. By usage of modern tools and to achieve good clearance in networking concepts by creating different Virtual Network Environment. This involves lectures, laboratories and assignments. It helped students to gain a better understanding of complex networking protocols and enable them to gain experience in creating and analyzing different network circumstances.

\section{References :}

[1] Attila Kuki "Modeling computer networks by the help of OpNet tools", Proceedings of the 9th International Conference on Applied Informatics Eger, Hungary, January 29-February 1, 2014.

[2] OPNET IT GURU: A Tool for Networking Education

[3] Jianyu Dong, Huiping Guo, "Enhance Computer Network Curriculum Using Collaborative Project-Based Learning", $120^{\text {th }}$ ASEE Annualal Conference and Exposition, Atlanta,USA, June 23-26, 2013.

[4] Te-Shun Chou1, Philip J. Lunsford1, and Keith, "Design of Simulation-based laborities for teaching Wireless Network Technologies", Proceedings of the Conference for Industry and Education Collaboration, Palm Springs, CA, USA, February 4-6, 2015.

[5] Michael W. Dixon and Terry W. Koziniec, "Using OpNet to Enhance Student Learning in Data Communications Course", Informing Science and Information Technology Education Conference (InSITE) 2002, 19 - 21 June 2002, Cork, Ireland

[6] OpNet Technologies, www.opnet.com 